\title{
Recurrent osteolytic lesions and subcutaneous fat necrosis in association with a developmental pancreatic cyst
}

\author{
P. HOLLINGWORTH, D. ISAACS, AND G. BYDDER \\ Northwick Park Hospital and Clinical Research Centre, Harrow, Middlesex
}

\begin{abstract}
SUMMARY A girl had repeated episodes of widespread osteolysis, subcutaneous fat necrosis, and periarticular fat necrosis mimicking arthritis. Her symptoms persisted for 4 years, after an initial episode of severe abdominal pain, and were accompanied by only minor abdominal symptoms. She was found to have a developmental pancreatic cyst (which was excised).
\end{abstract}

Disseminated fat necrosis is a rare complication of acute or chronic pancreatitis and pancreatic pseudocysts. It is usually an isolated rather than a recurrent event. This report describes a child with repeated osteolytic lesions, occurring over a 4-year period, in whom the diagnosis was ultimately made of a developmental pancreatic cyst.

\section{Case report}

A 7-year-old English girl was referred to this hospital in January 1977 with painful swollen fingers and toes. She had presented elsewhere shortly before her 5th birthday with swelling of the dorsum of the right foot. This had settled on antibiotics after drainage of a large amount of pus, but no organism had been isolated. One week later she had developed severe abdominal pain and fever, and at laparotomy free pus was present. This had again been sterile and no cause was found. A diagnosis of primary peritonitis had been made, antibiotics were continued, and she made a complete recovery.

After a further 3 months she had developed painful swelling of the dorsum of the left hand and of 2 toes on the left foot, with intermittent pyrexia. WBC was $22 \times 10^{9} / 1$, predominantly neutrophils but with an eosinophilia of $1.7 \times 10^{9} / 1$. Antibiotics had been given and the lesions resolved. Three weeks later she had developed a painless, hot swelling of the thigh which became fluctuant. $1 \mathrm{ml}$ of sterile fluid was aspirated. Immune deficiency was suspected but full investigations did not support this.

One year later, after a bout of diarrhoea, she had further pain and swelling of 2 toes and the terminal phalanges of all fingers of the right hand, and was referred to this hospital. On direct questioning it was found that all previous episodes had been accompanied by loose stools and she had had occasional bouts of abdominal pain, although these were not sufficiently severe for her to see a doctor.

$X$-rays of the affected areas showed lytic lesions breaching the cortex but without periostitis. The differential diagnosis initially was felt to be between leukaemia, sickle cell disease, histiocytosis X, multiple septic emboli, and other causes of osteolysis. Blood film showed no anaemia, no leucocytosis, and no abnormal cells, but slight eosinophilia. Haemoglobin electrophoresis was normal, as were neutrophil function tests. Antibiotics were withheld and the pain and swelling settled over a few weeks, with considerable recalcification radiologically (Fig. 1).

The girl remained well for a year, during which time she failed to attend follow-up appointments. She then presented, again after a bout of diarrhoea, with swelling of the hands and feet settling without

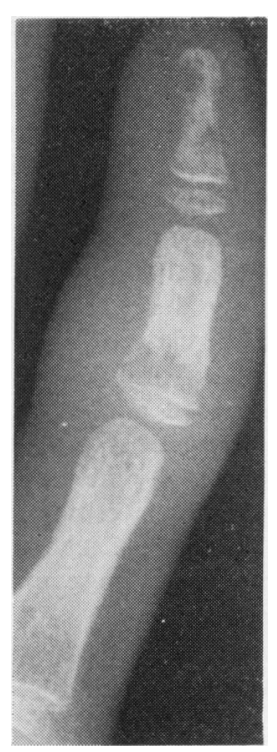

Fig. 1 Osteolytic lesions of the 5th finger January 1977. (12 months later $x$-ray showed disappearance of the lesions). 
treatment. Two weeks later she was admitted with swelling of one of the labia majora and subsequent panniculitis of the adjoining part of the thigh. A mass palpable in the left hypochondrium was thought to be the spleen. However barium meal showed a soft tissue mass in the region of the left kidney. Intravenous pyelogram and anterior ultrasound examination were both normal, but a CAT scan showed a cyst near the tail of the pancreas (Fig. 2). Serum amylase was $>2000$ Somogyi units $/ 100 \mathrm{ml}$ (normal $<200$ units $/ 100 \mathrm{ml}$ ). There was no history of abdominal trauma nor was there any suspicion of nonaccidental injury.

At laparotomy the pancreas appeared normal. A $6 \mathrm{~cm}$ cyst lay between the colon and the pancreas but separate from both. This was excised. Histology showed a cyst made of a fibrous wall, lined by mucous columnar cells. Within the wall were glands lined by mucous columnar cells, goblet cells, columnar cells with a brush border, and Paneth's cells. In addition, the wall contained pancreatic acini and islets of Langerhans. The appearances were of a pancreatic developmental cyst incorporating both intestinal and pancreatic tissue. Subsequently the patient has remained symptom-free.

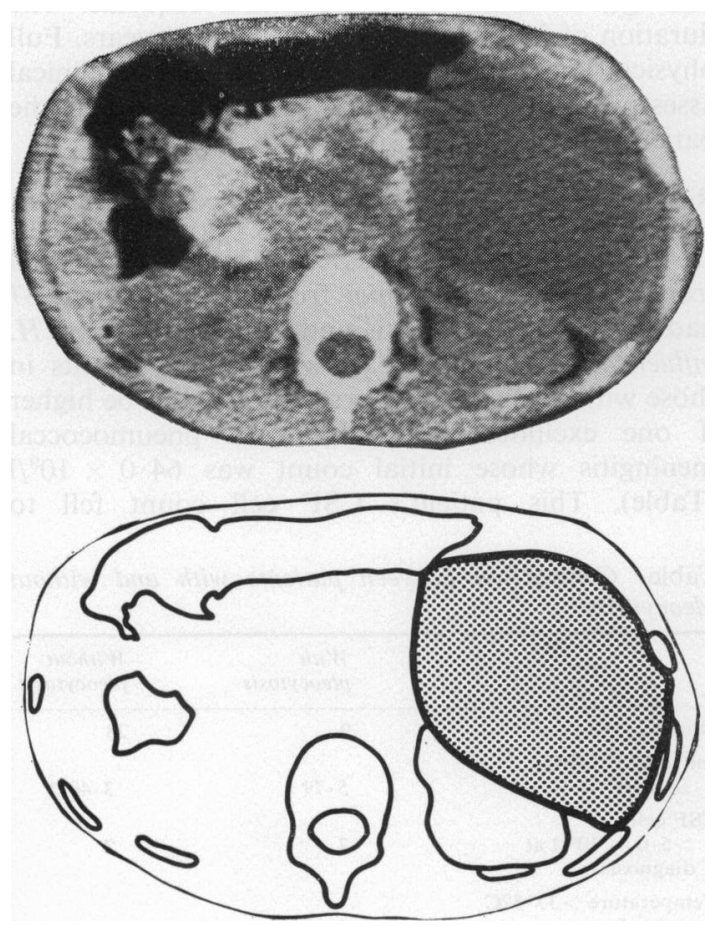

Fig. 2 CAT scan of the abdomen. Position and extent of cyst are shown in diagram.

\section{Discussion}

Although fat necrosis associated with pancreatic disease is usually intra-abdominal, disseminated fat necrosis may occur in the subcutaneous, periarticular, bone marrow or visceral fat. This is caused by pancreatic enzymes released into the circulation, and osteolysis may be secondary to necrosis of bone marrow or due to damage of small vessels by lipase (Achord and Gerle, 1966). Osteolytic lesions may be multiple and silent, but tend to be painful when affecting the small bones of the hands and feet. In children $x$-rays show punched-out lesions, sometimes with breach of the cortex and overlying periostitis, and radiological resolution may take months. The lesions are similar to the osteolytic lesions of metastatic neoplastic disease, sickle cell disease, and haematogenous osteomyelitis.

Subcutaneous fat necrosis presents as tender nodules while necrosis of periarticular fat may mimic arthritis. Fever, leucocytosis, and eosinophilia are common. Disseminated fat necrosis is a recognised complication of acute or chronic pancreatitis and pancreatic pseudocysts. When following acute pancreatitis the lesions may appear some weeks after apparent recovery (Shackleford, 1977).

Pancreatitis in childhood is being recognised with increasing frequency. Trauma is the most common cause, particularly handlebar injury, but child abuse should be excluded. Drugs, particularly corticosteroids and thiazide diuretics, may be responsible, and pancreatitis may be associated with mumps in which case it is invariably slight.

Pancreatic pseudocysts can follow acute pancreatitis and in most cases trauma has been implicated or suspected (Moossa, 1974). The child commonly presents some days or weeks after the injury with repeated vomiting or an 'acute abdomen'. A true cyst of the pancreas is said to be histologically distinguishable from a pseudocyst by the presence of an epithelial lining, but in practice the distinction is often difficult. True cysts are much less common than pseudocysts and may be neoplastic, hydatid, or congenital. Congenital cysts may be associated with mucoviscidosis or polycystic kidneys, or they may be developmental. The last are exceedingly rare (Miles, 1959), the few reported cases have presented with abdominal pain or swelling. In the case reported here the relationship between the patient's abdominal pain and her symptoms from disseminated fat necrosis was obscure. Measurement of serum amylase might have enabled the diagnosis to have been made earlier.

We thank Lord Smith of Marlow, Dr S. M. Tucker, and Dr B. M. Ansell for permission to report this patient. 


\section{References}

Achord, J. L., and Gerle, R. D. (1966). Bone lesions in pancreatitis. American Journal of Digestive Diseases, 11, 453-459.

Miles, R. N. (1959). Pancreatic cyst in the newborn. Annals of Surgery, 149, 576-581.

Moossa, A. R. (1974). Pancreatic pseudocysts in children.
Journal of the Royal College of Surgeons of Edinburgh, 19, 149-158.

Shackleford, P. G. (1977). Osseous lesions and pancreatitis. American Journal of Diseases of Children, 131, 731-732.

Correspondence to $\mathrm{Dr}$ P. Hollingworth, Mathilda and Terence Kennedy Institute of Rheumatology, Bute Gardens, London W6 7DW.

\section{Lumbar punctures, meningitis, and persisting pleocytosis}

\section{KEVIN D. CONNOLLY}

\section{Children's Research Centre, Our Lady's Hospital for Sick Children, Dublin}

SUMMARY A retrospective survey of children with haemophilus and pneumococcal meningitis was carried out to assess the significance of persistent CSF pleocytosis and the need for repeat lumbar punctures after adequate treatment. Persistent pleocytosis was noted in 9 of 27 patients with haemophilus meningitis; this tended to be present in those with higher initial CSF white blood counts and lower initial CSF glucose contents. No sequelae were noted in those with persistent pleocytosis. Repeat lumbar punctures were not of clinical benefit and tended to result in longer treatment which was not warranted.

One criterion used to decide when to stop treatment in a child with bacterial meningitis is the presence of a CSF white blood count of $<0.050 \times 10^{9} / 1$ after 10-14 days of treatment (Smith et al., 1973). However, not only may a lumbar puncture be dangerous (Shinefield, 1975), but children who have received apparently adequate treatment can show persistent increases in the CSF white blood count. This survey was carried out to assess the significance of persistent pleocytosis and the need for a repeat lumbar puncture in a clinically well child after reasonable treatment.

\section{Patients and methods}

Records of all patients aged over 2 months admitted to this hospital between January 1972 and July 1977 with a diagnosis of bacterial meningitis were examined. The survey was confined to those who grew Haemophilus influenzae or Streptococcus pneumoniae from their CSF. Patients with recognised anatomical defects of skull or spine, those with immunological disorders, and those who did not have lumbar punctures repeated between days 10 and 14 were excluded. Those with meningococcal meningitis were also excluded as very few have persistent pleocytosis and their prognosis is better.
All those with $H$. influenzae received chloramphenicol for at least 10 days. In addition 22 received ampicillin for 10 days. 13 were given sulphonamide and 5 were given penicillin for varying periods. All with pneumococcal meningitis received penicillin for at least 10 days. In addition, 6 were given chloramphenicol, 6 were given sulphonamide, and 4 were given ampicillin for varying periods. Persistent pleocytosis was defined as a CSF white blood count $>0.050 \times 10^{9} / 1$ between days 10 and 14 .

37 babies fulfilling these criteria were examined with regard to organism, initial and repeat CSF findings, duration of pyrexia, and sequelae. The duration of follow-up was from $1 \frac{1}{2}$ to $5 \frac{1}{2}$ years. Full physical and neurological examination and clinical assessment of hearing were carried out when the patients were recalled.

\section{Results}

Of the 37 patients studied, $H$. influenzae was isolated from 27, and $S$. pneumoniae from 10 . Nine of the 37 had persistent pleocytosis and all of these grew $H$. influenzae. The initial CSF white blood counts in those with persistent pleocytosis tended to be higher if one excludes one baby with pneumococcal meningitis whose initial count was $64.0 \times 10^{9} / 1$ (Table). This patient's CSF cell count fell to

Table Comparison between patients with and without pleocytosis

\begin{tabular}{lll}
\hline & $\begin{array}{l}\text { With } \\
\text { pleocytosis }\end{array}$ & $\begin{array}{l}\text { Without } \\
\text { pleocytosis }\end{array}$ \\
\hline $\begin{array}{l}\text { Number } \\
\begin{array}{l}\text { Initial CSF WBC } \\
\quad \times 10^{9} / 1\end{array}\end{array}$ & 9 & 28 \\
$\begin{array}{l}\text { CSF cells } \\
\quad 5.0 \times 10^{9} / 1 \text { at } \\
\text { diagnosis }\end{array}$ & $5 \cdot 79$ & $3 \cdot 402^{*}$ \\
$\begin{array}{l}\text { Temperature }>37 \cdot 5^{\circ} \mathrm{C} \\
\quad \text { at day 5 }\end{array}$ & 7 & 7 \\
Sequelae & 5 & \\
\hline
\end{tabular}

*One patient with CSF WBC of $64.0 \times 10^{9} / 1$ is excluded. 